Research Paper

\title{
The survival benefit of intensified full-dose XELOX chemotherapy concomitant to radiotherapy and then resting-period consolidation chemotherapy in locally advanced rectal cancer
}

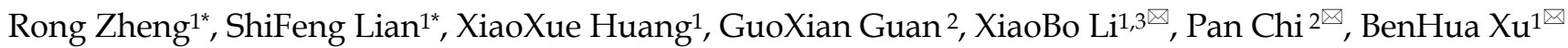 \\ 1. Department of Radiation Oncology, Fujian Medical University Union Hospital, Fuzhou, Fujian 350001, P.R. China \\ 2. Department of Colorectal Surgery, Fujian Medical University Union Hospital, Fuzhou, Fujian 350001, P.R. China \\ 3. Collogy of medical technology and engineering, Fujian Medical University, Fuzhou 350001, P.R. China \\ "These authors contributed equally to this work \\ $\square$ Corresponding author: BenHua Xu, E-mail: benhuaxu@163.com; Pan Chi, E-mail: cp3169@163.com; XiaoBo Li, E-mail: lixiaobo2004@126.com \\ (c) Ivyspring International Publisher. This is an open access article distributed under the terms of the Creative Commons Attribution (CC BY-NC) license \\ (https://creativecommons.org/licenses/by-nc/4.0/). See http://ivyspring.com/terms for full terms and conditions.
}

Received: 2018.07.02; Accepted: 2018.11.01; Published: 2019.01.01

\begin{abstract}
Purpose: To evaluate the effect of an intensified capecitabine and oxaliplatin (XELOX) chemoradiation treatment followed by one cycle of consolidation chemotherapy before surgery in locally advanced rectal cancer (LARC).

Methods and Materials: Patients with histologically confirmed, newly diagnosed, locally advanced rectal adenocarcinoma (cT3-T4 and/or $\mathrm{cN}+$ ) were enrolled. All patients received 3-dimensional conformal radiotherapy (3D-CRT) or intensity-modulated radiotherapy (IMRT) with a dose 50.4Gy in 25 fraction, with two cycles of concurrent XELOX chemotherapy. Thereafter, another cycle of consolidation chemotherapy with XELOX/FLOFOX was administered during the resting period after completion of concurrent chemoradiation (CRT). Tumor response, toxicities, surgical complications, and long-term clinical outcomes were recorded.

Results: From January 2011 to December 2013, a total of 96 patients were enrolled in the study. All patients completed the treatment plan of concurrent chemoradiation and consolidate chemotherapy. During concurrent chemoradiation, the incidence of grade $3 / 4$ toxicities was leucopenia $(2.1 \%)$, thrombocytopenia (4.2\%), diarrhea (6.3\%). 18 patients (18.8\%) developed surgical complications. Pathologic complete response (pCR) was achieved in 20 (20.8\%) patients. Tumor down-staging occurred in $69(71.9 \%)$ patients and down-staging of nodes occurred in 47 (49.0\%) patients. Of these 96 patients, 5 -year local recurrence-free survival, metastasis-free survival, disease-free survival and overall survival rates was $98.9 \%, 84.7 \%, 83.7 \%$ and $82.1 \%$, respectively, with a median follow-up of 4.24 years.

Conclusions: The intensified treatment paradigm of XELOX concurrent chemoradiation followed by one cycle of consolidation chemotherapy was well tolerated in our cohort and provided a promising long-term oncologic outcome, which warranted further investigation in a randomize trails.
\end{abstract}

Key words: rectal cancer; XELOX; chemoradiation

\section{Introduction}

In locally advanced rectal cancer (LARC), fluorouracil based preoperative concurrent chemoradiation (CRT) followed by total mesorectal excision (TME) and adjuvant chemotherapy is the standard therapy modality ${ }^{[1,2]}$. With this triple treatment modality, patients had a good local and regional control rate around $95 \%[1-4]$, however, the distant metastasis risk still high to $30 \%{ }^{[2-4]}$. In theory, intensified chemotherapy before or after TME can reduce the risk of distant metastasis. 
However, the standard preoperative treatment contains single chemotherapy drug, either fluorouracil or capecitabine, aiming to sensitize radiotherapy effect, and consequently, lower the radiation therapy dose to 45Gy of pelvis and 50.4Gy of the primary site. Theoretically, insufficient preoperative chemotherapy and locally pelvis radiotherapy may control the primary tumor and pelvic disease well, but is not enough to eliminate the potential distant metastasis disease. So, change the radiosensitizition concurrent chemotherapy regimen to a full dose curative regimen may reduce the high distant metastasis risk.

There is no anti-tumor treatment during the 6 to 8 weeks' interval time for waiting surgery, which may cause many questions. An in-vitro study showed that in the later period of radiotherapy, the Tpot of tumor will be returned to the pretreatment levels ${ }^{[5]}$. And Jeong et al[ ${ }^{6]}$ found that if the interval time prolonged more than 8 weeks, the distant metastasis risk will increase to $24 \%$. So, adding some intensified full-dose chemotherapy during this resting period may be useful.

Oxaliplatin is an effective drug, which can be combined with fluorouracil to improve the survival of colon cancer patients[7-9]. However, several prospective trials ${ }^{[10-12]}$ failed to verify the benefit of adding oxaliplatin to 5-Fu as preoperative CCRT in stage II/III rectal cancer. The potential reason may be partly explained by the weekly low dose administered of oxaliplatin, which mainly aims to increase radiosensitivity.

Therefore, we design this study which contains 2 cycles of full dose XELOX concurrent with radiotherapy and 1 cycle of consolidation chemotherapy with XELOX/FLOFOX during the interval time trying to improve the distant metastasis free survival (DMFS) and overall survival (OS).

\section{Material and methods}

\section{Eligibility criteria}

From January 2011 to December 2013, a total of 96 patients with histologically confirmed, newly diagnosed LARC (cT3-T4 and/or $\mathrm{N}+$ nonmetastatic rectal adenocarcinoma) located within $12 \mathrm{~cm}$ of the anal verge were enrolled. All patients met the following criteria: Karnofsky Performance Status (KPS) $\geq 70 \geq 70$, potentially resectable diseases, adequate hematologic function (leukocyte count $>4 \times 10^{9} / \mathrm{L}$ and platelet count $>100 \times 10^{9} / \mathrm{L}$ ), adequate renal and hepatic function (creatinine clearance $>60 \mathrm{~mL} / \mathrm{min}$ and AST $\leq 100 \mathrm{U} / \mathrm{L}$ ). Patients suffering from the following conditions will be excluded: with heart, liver or renal dysfunction, a history of previous malignancy, previous treatment with chemotherapy or radiotherapy, pregnancy or breast-feeding, or presence of a psychiatric disorder. The study protocol was approved by Institutional Review Board of The Affiliated Union Hospital of Fujian Medical University (Fuzhou, China) and all patients provided written informed consent.

Within two weeks before initiation of chemoradiation, all patients were evaluated with a complete history and physical examination, including digital rectal examination, complete blood count, hepatic and renal function tests, colonoscopy and biopsy, computed tomography (CT) of the thorax, magnetic resonance imaging (MRI) of the abdominal-pelvic and endorectal ultrasound. Clinical stage was assessed according to the $7^{\text {th }}$ edition of the AJCC Cancer Staging Manual[13] based on the radiographic evidence mentioned above.

\section{Treatment protocol}

\section{Radiotherapy}

Radiotherapy was delivered according to the previously reports ${ }^{[14}$ 15], either 3-dimensional conformal radiotherapy (3D-CRT) or intensity modulated radiotherapy (IMRT) was used according to the patient's medical insurance. Planning target volume (PTV) was defined as an additional $1.0 \mathrm{~cm}$ beyond the scope of the clinical target volume (CTV) and the gross tumor volume (GTV). Briefly, the 3D-CRT was delivered by two lateral fields with $15 \mathrm{MV}$ and one posterior field with $6 \mathrm{MV}$ and a dose of 45Gy in 25 fractions to CTV with 5.4 Gy in 3 fractions boosted to GTV. As for IMRT, GTV was determined from digital rectal examination, endoscopy ultrasound, CT and MRI. And CTV was including the entire mesorectum, presacral, and internal iliac lymph node drainage regions with at least a $3 \mathrm{~cm}$ craniocaudal margin to the GTV. Patients with IMRT were delivered through five fields $(6 \mathrm{MV})$ with a dose of 45 Gy to the CTV and 50.4 Gy to the GTV in 25 fractions.

\section{Chemotherapy}

During the course of radiation therapy, all patients were conducted with 2 cycles of XELOX (oxaliplatin $130 \mathrm{mg} / \mathrm{m}^{2}$ on day 1 plus capecitabine 825 $\mathrm{mg} / \mathrm{m}^{2}$ twice daily from day 1-14 for every 21 days). Patients received another cycle of consolidation chemotherapy with XELOX/FLOFOX one week after CRT. The consolidation XELOX consisted of oxaliplatin $\left(130 \mathrm{mg} / \mathrm{m}^{2}\right.$ on day 1$)$ and capecitabine $\left(1000 \mathrm{mg} / \mathrm{m}^{2}\right.$, twice per day from day 1 to day 14) for every 21 days. And the FOLFOX consisted of folinic acid $\left(400 \mathrm{mg} / \mathrm{m}^{2}\right)$, oxaliplatin $\left(85 \mathrm{mg} / \mathrm{m}^{2}\right)$ on day 1 , 
bolus fluorouracil $400 \mathrm{mg} / \mathrm{m} 2$ on day 1-2, and a $46-\mathrm{h}$ infusion of fluorouracil $1200 \mathrm{mg} / \mathrm{m}^{2}$ repeated every 3 weeks.

\section{Surgery and histopathology}

Total mesorectal excision was scheduled 6-8 weeks after the completion of chemoradiation (3-5 weeks after the final cycle). Pathological stage was determined on the post-CRT surgical specimen, and histological regression grade was performed according to the Wheelers' classification ${ }^{[16]}$. A pCR (ypT0N0) was defined as the complete absence of tumor cells at the primary site and without lymph node involved. Postoperative adjuvant chemotherapy was administered at the surgeon's discretion.

\section{Statistical analysis}

The primary endpoint was $\mathrm{pCR}$ rate. The other protocol-defined efficacy endpoints included local recurrence-free survival (LFS), metastasis-free survival (MFS), disease-free survival (DFS) and overall survival rates(OS). Continuous variable was presented as medians and ranges and categorical data was presented as numbers and percentages. Survival time was between the beginning of CRT to the date of event or the last follow-up, and Kaplan-Meier method was used to calculate the survival curves. All statistical assessments were 2 -sided and a $P$ values less than 0.05 were considered significant. Statistical analyses were performed with SPSS 23.0 (SPSS Inc, Chicago, IL, USA).

\section{Results}

From January 2011 to December 2013, we enrolled 96 patients with newly diagnosed T3/4 and/or $\mathrm{N}+$ rectal cancer. The number of patients in clinical T stage of T2, T3 and T4 was $6(6.3 \%), 37$ (38.5\%)and $53(55.2 \%)$ respectively.76 patients were detected with lymph node involvement. 67 patients (69.7\%) had a tumor located $\leq 5 \mathrm{~cm}$ from the anal verge. All the patients completed the full course of XELOX concurrent chemoradiation and the resting consolidation chemotherapy of XELOX/FOLFOX. The median interval time from the end of radiotherapy to surgery was 55 days. And the median follow-up time was 4.24 years. Table 1 summarizes the characteristics of the 96 patients who were ultimately enrolled.

Table 2 summarizes the treatment-related adverse events. During the CRT period, the most frequently occurring grade $3 / 4$ hematological toxicity was Thrombocytopenia (4.2\%), and the most common non-hematological adverse event was diarrhea (6.3\%). As for the surgical resection or postoperative complication, one or more adverse events occurred in
$18(18.8 \%)$ of patients, including $2(2.1 \%)$ anastomotic fistula, $3(3.1 \%)$ pulmonary infection, $3(3.1 \%)$ urinary tract infection, 11(11.5) infection of incision, and 3 (3.1\%) intestinal obstruction.

Table 1. Demographic and clinical features for all patients

\begin{tabular}{ll}
\hline Variable & value \\
\hline Patients number & 96 \\
Age (range) & $54.93(29,69)$ \\
Gender & \\
Male & 66 \\
Female & 30 \\
Distance from anal verge & \\
Low $(0-5 \mathrm{~cm})$ & 67 \\
Middle(5.1-10cm) & 28 \\
High(10.1-12cm) & 1 \\
Pretreatment CEA(range) & $16.90(0.4,427.8)$ \\
Pretreatment CA19-9(range) & $42.70(0.6,721.20)$ \\
cT & \\
cT1 & 0 \\
cT2 & 6 \\
cT3 & 37 \\
cT4 & 53 \\
cN- & 20 \\
cN+ & 76 \\
Radiation & \\
3D-CRT & 57 \\
IMRT & 39 \\
Surgery & \\
LAR & 75 \\
APR & 15 \\
Hartmann & 6 \\
Median time between CRT and surgery(range) & $55(35,91)$ \\
Median number of resected nodes & $12(0-34)$ \\
Median fellow-up time(year) & $4.24(0.53,6.02)$ \\
\hline
\end{tabular}

Table 2. Adverse Events (AEs) in rectal cancer patients treated with intensive neoadjuvant chemoradiotherapy

\begin{tabular}{ll}
\hline AEs & No. of patients $(\%)$ \\
\hline Grade 3-4 toxicity during CRT & \\
Anemia & $0(0 \%)$ \\
Leucopenia & $2(2.1 \%)$ \\
Thrombocytopenia & $4(4.2 \%)$ \\
Diarrhea & $6(6.3 \%)$ \\
AEs of surgery & \\
At least one adverse event & $18(18.8 \%)$ \\
Anastomotic fistula & $2(2.1 \%)$ \\
Pulmonary infection & $3(3.1 \%)$ \\
Urinary tract infection & $3(3.1 \%)$ \\
Incision infection & $11(11.5 \%)$ \\
Intestinal obstruction & $3(3.1 \%)$ \\
\hline
\end{tabular}

Tumor downstaging occurred in 69 (71.9\%) patients and lymph node down-staging in 47 (49.0\%) patients. Wilcoxon's signed-rank test indicated a significant difference in tumor stage before treatment and after surgery $(\mathrm{P}<0.001)$. Table 3 shows the tumor downstaging. According to the predefined criteria, the pCR (ypT0N0) rate was $20.8 \%$. The rectal cancer regression grade (RCRG) stage was Grade 1 in 56 (58.3\%) patients, Grade 2 in 25 (35.5\%) patients, Grade 3 in $6(5.2 \%)$ patients. Table 4 shows the pathological features.

With a median follow-up of 4.24 years (range, 0.53-6.02 years), 1 patient was diagnosed with 
recurrence, 15 patients were confirmed with distant metastasis ( 6 cases in lung, 1 case in lung and liver, 4 cases in liver, 1 case in lung brain and liver, 1 case in bone, 2 cases in brain). 15 patients died in the whole cohort. The 5-year local recurrence-free survival, metastasis-free survival, disease-free survival and overall survival rates were $98.9 \%$ (95\% CI: $97.8 \%$, 100\%), 84.7\% (95\% CI: 80.9\%, 88.5\%), 83.7\% (95\% CI: $79.8 \%, 87.6 \%$ ) and $82.1 \%$ (95\% CI: $77.2 \%, 86.9 \%)$, respectively. Figure 1 shows the 5-year LFS, MFS, DFS and OS rates of all 96 patients.

Table 3. Correlation between clinical and pathological $\mathrm{T}$ and $\mathrm{N}$ stage

\begin{tabular}{lllllllll}
\hline & \multicolumn{3}{c}{ Pathological T staging } & \multicolumn{3}{c}{ Pathological N staging } \\
\hline & ypT0 & ypT1 & ypT2 & ypT3 & ypT4 & ypN0 & ypN1 & ypN2 \\
\hline cT2 & 3 & 1 & 1 & 1 & 0 & 6 & 0 & 0 \\
cT3 & 6 & 2 & 10 & 18 & 1 & 22 & 12 & 3 \\
cT4 & 12 & 4 & 10 & 21 & 6 & 34 & 16 & 3 \\
cN0 & 6 & 3 & 3 & 7 & 1 & 15 & 4 & 1 \\
cN+ & 15 & 4 & 18 & 33 & 6 & 47 & 24 & 5 \\
\hline
\end{tabular}

\section{Discussion}

Neoadjuvant chemoradiation followed by TME surgery had been the standard care for LARC. However, most of phase III clinical trials ${ }^{[1-3,17]}$ failed to show the addition of CRT before TME surgery could improve survival, which suggests the continued
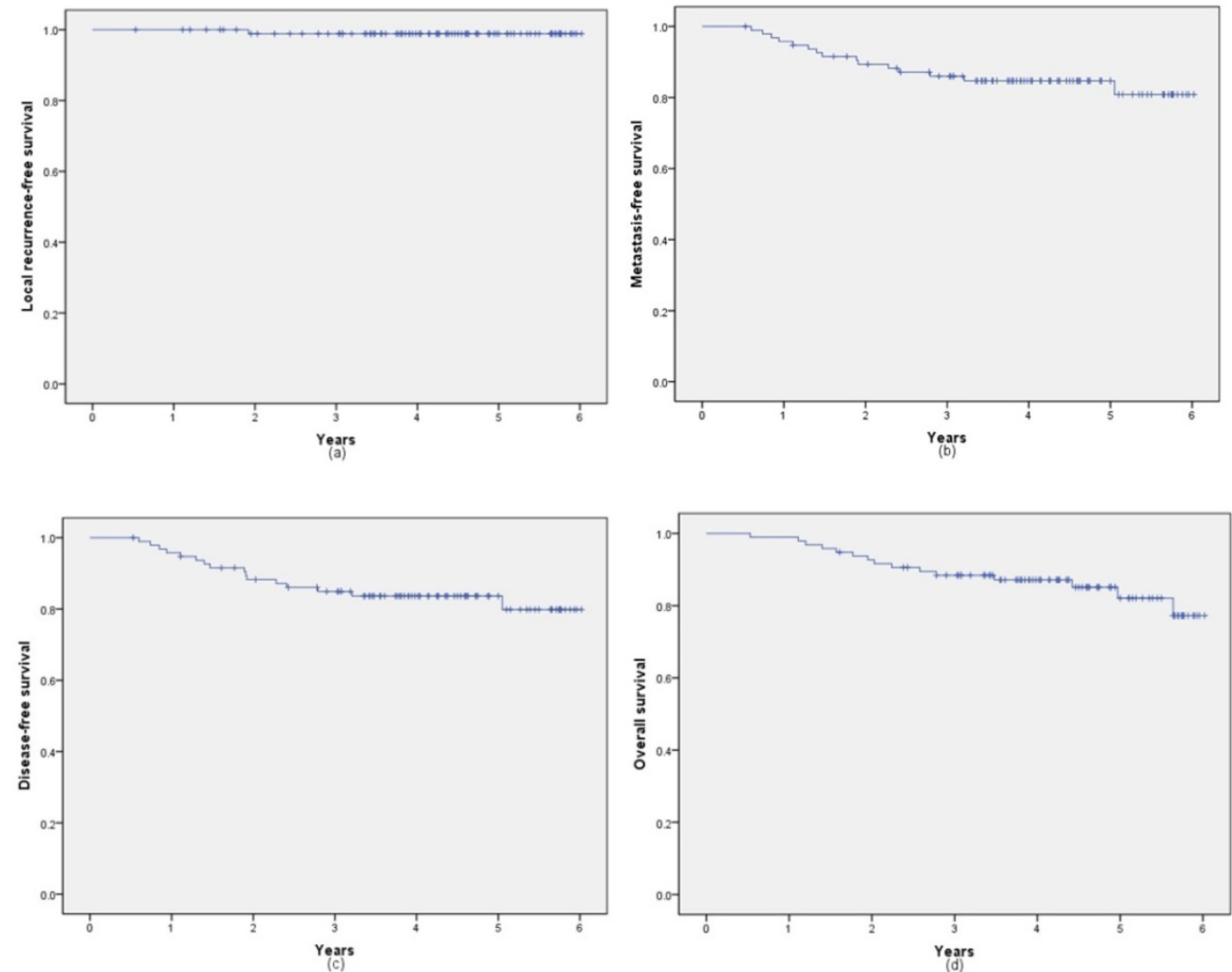

Figure 1. Kaplan-Meier analysis of local recurrence-free survival (a), metastasis-free survival (b), disease-free survival (c) and overall survival rates (d). 
In the setting of neoadjuvant chemoradiation, the optimal sequencing of preoperative CRT and CT before resection of rectal cancer had been studied in trails, either the form of neoadjuvant chemotherapy (NAC) followed by CRT, or CRT followed by NAC. As for the approach of NAC followed by CRT, there were lots of studies. However, in the trail of GCR-3[18], it failed to show an improvement either DFS or OS at 5 years of the neoadjuvant arm. As a result, the strategy of NAC followed by CRT seems to be unsatisfactory.

Unlikely, the strategy of CRT followed by NAC is less well studied but shown with promising results. Zhu et al. ${ }^{[19]}$ studied the effect of one additional cycle of capecitabine after XELOX CRT in LARC. They reported a pCR rate of $15.8 \%$, with acceptable toxicity and tolerability. Bujko et al.[20] also reported a pCR rate of $16 \%$ in the arm of SC-RT/NAC (RT followed 3 cycles of FOLFOX4). Garcia-Aguilar et al. [21] studied the effect of 0-6 additional cycles of modified FOLFOX6 chemotherapy after CRT. They reported the $\mathrm{pCR}$ rates increased significantly with increasing cycles of additional FOLFOX6, with a pCR rate of $38 \%$ for patients with 6 cycles of additional FOLFOX6, compared with an $18 \%$ pCR rate for patients who did not receive. In our study, we achieved a higher $\mathrm{pCR}$ rate of $20.8 \%$, which was similar to that of the previous studies. When taken the RCRG1 rate of $58.3 \%$ into consideration, the good response rate to this treatment strategy was fairly high. These results indicated that the intensified full-dose CRT followed by one cycle of resting-period consolidation chemotherapy worked for most of the patients in our cohort.

Patients with pathologic $C R$ were shown to correlate with better local control and improved survival. However, it had never demonstrated that a pCR following neoadjuvant therapy for rectal cancer meets formal criteria as a surrogate endpoint for LC or OS[22, 23]. While, previous studies generally focused on short-term pathologic endpoints, such as pCR rates. The long-term oncological outcomes after TNT lacked of reports. In the study by Zhu et al. ${ }^{[19]}$, they reported a 3-year DFS and OS rates of $57.4 \%$ and $66.0 \%$, respectively. Bujko et al.[20] reported a 3-year DFS and OS rates of $53 \%$ and $73.0 \%$ in the arm of SC-RT/NAC. Being similarly with the results of CRT followed by NAC, the long-term outcome of 5-year DFS or OS is around with $60 \%$ and $75 \%$ in the strategy of NAC followed by CRT[18, 24, 25]. To current study, we achieved a 5-year DFS, OS of $84.7 \%$ and $81.1 \%$ respectively, which were superior to previous studies.

In theory, an increased dose of chemotherapy could be accompanied by increased toxicities. However, the treatment paradigm with an intensified chemotherapy in our study achieved better long-term oncological outcomes without increasing the toxicities, or surgical complications when compared to previous studies $[1,2,12,19,26-29]$. Some reasons as bellows may be partly explained for this result.

Firstly, with the XELOX regimen for concurrent chemotherapy, we administrated capecitabine from day 1 to day 14 with a high dose of oxaliplatin every 3 weeks. With this kind of CRT strategy, there was one week free out of chemotherapy. During this period, patients could recover from the side effects and increase the treatment compliance. So, most patients could receive a full dose of chemotherapy and radiation as planned without increased toxicity.

One may argue that, adding oxaliplatin concomitant to radiotherapy was not proved to improve survival. However, in most of the previous studies, the oxaliplatin was administrated in a low dose by weekly as a radiosensitizer. In our studies, oxaliplatin was administrated in a full dose by a 3-weekly way. This kind of high dose oxaliplatin in $X E L O X$ regimen was proved to be effective in both palliative and adjuvant settings. With the high dose of oxaliplatin, we could expose micrometastasis to an intensified chemotherapy and achieved a favorable long-term outcome.

Furthermore, the relatively high pCR and better survival rates could also be the results of adding an intensified consolidate chemotherapy during the resting period before surgery. Studies[30, 31] had observed that a significant proportion of patients developed recuperation of tumor metabolism during the resting period after CRT, and it may contribute to the therapy failure of distance metastasis. When adding a consolidate chemotherapy, the risk of developing tumor reputation is decreasing. In our study, we added another intensified resting-period XELOX/FOLFOX chemotherapy, which exposed the systemic micrometastasis and local irradiated tumor cells to full dose chemotherapy, may ultimately contribute to the improvement survival benefits, especially for patients with longer waiting period. The dates in our study can provide theoretical basis support for the design of randomized controlled trials in the future, and it is expected to further optimize the preoperative radiotherapy treatment plan in the interval time for waiting surgery in rectal cancer patients.

Our study is subjected to several limitations. First, this study was not a randomized controlled study. When compared our results with other similar studies should be cautious. Second, all the patients were from a single institution and the results may not be generalized to other populations, which means our data may not be insufficient to argue and to be 
conclusive about these results. However, the long-term survival result provided by our study is promising. And the treatment paradigm of intense full-dose XELOX concurrent chemoradiation followed by consolidation chemotherapy deserved to be further researched in a multi-center randomize trails.

\section{Conclusion}

In conclusion, results from our study indicate that a full-dose of XELOX chemoradiation followed by one cycle of resting-period consolidation chemotherapy before surgery has a good treatment efficacy with tolerated toxicities. And this treatment paradigm provided a promising long-term oncologic outcome warrant further investigation in a randomize trails.

\section{Acknowledgements}

\section{Ethics approval and consent to participate}

This study was operated under Fujian Medical University Union Hospital Ethics Committee approval. Patients were consented at the time of inclusion.

\section{Authors' contributions}

Rong Zheng: responsibility for the first draft of the manuscript.

ShiFeng Lian: responsibility for the first draft of the manuscript.

XiaoXue Huang: proportion of patients entered by an individual.

GuXian Guan: undertook the collection of data.

Pan Chi: undertook the collection of data.

BenHua Xu: the original idea for the research.

XiaoBo Li: Provide support of radiation oncology physics.

\section{Funding}

This study was supported by Fujian provincial health and family planning research talent training program (Grant number: 2017-ZQN-30).

\section{Competing Interests}

The authors have declared that no competing interest exists.

\section{References}

1. Sauer R, Becker H, Hohenberger W, Rodel C, Wittekind C, Fietkau R, et al. Preoperative versus postoperative chemoradiotherapy for rectal cancer. N Engl J Med. 2004; 351: 1731-40.

2. Bosset JF, Collette L, Calais G, Mineur L, Maingon P, Radosevic-Jelic L, et al. Chemotherapy with preoperative radiotherapy in rectal cancer. N Engl J Med. 2006; 355: 1114-23

3. Peeters KC, Marijnen CA, Nagtegaal ID, Kranenbarg EK, Putter H, Wiggers T, et al. The TME trial after a median follow-up of 6 years: increased local control but no survival benefit in irradiated patients with resectable rectal carcinoma. Ann Surg. 2007; 246: 693-701.

4. Gollins S, Sebag-Montefiore D. Neoadjuvant Treatment Strategies for Locally Advanced Rectal Cancer. Clin Oncol (R Coll Radiol). 2016; 28: 146-51.
5. Murata R, Nishimura Y, Shibamoto Y, Hiraoka M, Abe M. Changes in cell proliferative parameters of SCCVII and EMT6 murine tumors after single-dose irradiation. Jpn J Cancer Res. 1996; 87: 662-8.

6. Jeong DH, Lee HB, Hur H, Min BS, Baik SH, Kim NK. Optimal timing of surgery after neoadjuvant chemoradiation therapy in locally advanced rectal cancer. J Korean Surg Soc. 2013; 84: 338-45.

7. Morse MA. Adjuvant therapy of colon cancer: current status and future developments. Clin Colon Rectal Surg. 2005; 18: 224-31.

8. Andre T, Boni C, Mounedji-Boudiaf L, Navarro M, Tabernero J, Hickish T, et al. Oxaliplatin, fluorouracil, and leucovorin as adjuvant treatment for colon cancer. N Engl J Med. 2004; 350: 2343-51.

9. Andre T, Boni C, Navarro M, Tabernero J, Hickish T, Topham C, et al. Improved overall survival with oxaliplatin, fluorouracil, and leucovorin as adjuvant treatment in stage II or III colon cancer in the MOSAIC trial. J Clin Oncol. 2009; 27: 3109-16

10. Rodel C, Liersch T, Becker H, Fietkau R, Hohenberger W, Hothorn T, et al. Preoperative chemoradiotherapy and postoperative chemotherapy with fluorouracil and oxaliplatin versus fluorouracil alone in locally advanced rectal cancer: initial results of the German $\mathrm{CAO} / \mathrm{ARO} / \mathrm{AIO}-04$ randomised phase 3 trial. Lancet Oncol. 2012; 13: 679-87.

11. Gerard JP, Azria D, Gourgou-Bourgade S, Martel-Laffay I, Hennequin C, Etienne PL, et al. Comparison of two neoadjuvant chemoradiotherapy regimens for locally advanced rectal cancer: results of the phase III trial ACCORD 12/0405-Prodige 2. J Clin Oncol. 2010; 28: 1638-44.

12. Aschele C, Cionini L, Lonardi S, Pinto C, Cordio S, Rosati G, et al. Primary tumor response to preoperative chemoradiation with or without oxaliplatin in locally advanced rectal cancer: pathologic results of the STAR-01 randomized phase III trial. J Clin Oncol. 2011; 29: 2773-80.

13. Edge SB, Compton CC. The American Joint Committee on Cancer: the 7th edition of the AJCC cancer staging manual and the future of TNM. Ann Surg Oncol. 2010; 17: 1471-4.

14. Chen YG, Chen MQ, Guo YY, Li SC, Wu JX, Xu BH. Apparent Diffusion Coefficient Predicts Pathology Complete Response of Rectal Cancer Treated with Neoadjuvant Chemoradiotherapy. PLoS One. 2016; 11: e0153944.

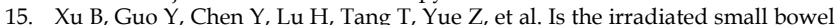
volume still a predictor for acute lower gastrointestinal toxicity during preoperative concurrent chemo-radiotherapy for rectal cancer when using intensity-modulated radiation therapy? Radiat Oncol. 2015; 10: 257.

16. Wheeler JM, Warren BF, Mortensen NJ, Ekanyaka N, Kulacoglu H, Jones AC, et al. Quantification of histologic regression of rectal cancer after irradiation: a proposal for a modified staging system. Dis Colon Rectum. 2002; 45: 1051-6.

17. van Gijn W, Marijnen CA, Nagtegaal ID, Kranenbarg EM, Putter H, Wiggers T, et al. Preoperative radiotherapy combined with total mesorectal excision for resectable rectal cancer: 12-year follow-up of the multicentre, randomised controlled TME trial. Lancet Oncol. 2011; 12: 575-82.

18. Fernandez-Martos C, Garcia-Albeniz X, Pericay C, Maurel J, Aparicio J, Montagut $C$, et al. Chemoradiation, surgery and adjuvant chemotherapy versus induction chemotherapy followed by chemoradiation and surgery: long-term results of the Spanish GCR-3 phase II randomized trialdagger. Ann Oncol. 2015; 26: 1722-8.

19. Zhu J, Gu W, Lian P, Sheng W, Cai G, Shi D, et al. A phase II trial of neoadjuvant IMRT-based chemoradiotherapy followed by one cycle of capecitabine for stage II/III rectal adenocarcinoma. Radiat Oncol. 2013; 8: 130.

20. Bujko K, Wyrwicz L, Rutkowski A, Malinowska M, Pietrzak L, Krynski J, et al. Long-course oxaliplatin-based preoperative chemoradiation versus $5 \times 5$ Gy and consolidation chemotherapy for cT4 or fixed cT3 rectal cancer: results of a randomized phase III study. Ann Oncol. 2016; 27: 834-42.

21. Garcia-Aguilar J, Chow OS, Smith DD, Marcet JE, Cataldo PA, Varma MG, et al. Effect of adding mFOLFOX6 after neoadjuvant chemoradiation in locally advanced rectal cancer: a multicentre, phase 2 trial. Lancet Oncol. 2015; 16: 957-66.

22. Petrelli F, Borgonovo K, Cabiddu M, Ghilardi M, Lonati V, Barni S. Pathologic complete response and disease-free survival are not surrogate endpoints for 5-year survival in rectal cancer: an analysis of 22 randomized trials. J Gastrointest Oncol. 2017; 8: 39-48.

23. Prentice RL. Surrogate endpoints in clinical trials: definition and operational criteria. Stat Med. 1989; 8: 431-40.

24. Sclafani F, Brown G, Cunningham D, Wotherspoon A, Tait D, Peckitt C, et al. PAN-EX: a pooled analysis of two trials of neoadjuvant chemotherapy followed by chemoradiotherapy in MRI-defined, locally advanced rectal cancer. Ann Oncol. 2016; 27: 1557-65.

25. Schou JV, Larsen FO, Rasch L, Linnemann D, Langhoff J Hogdall E, et al. Induction chemotherapy with capecitabine and oxaliplatin followed by chemoradiotherapy before total mesorectal excision in patients with locally advanced rectal cancer. Ann Oncol. 2012; 23: 2627-33.

26. Gao YH, Zhang X, An X, Cai MY, Zeng ZF, Chen G, et al. Oxaliplatin and capecitabine concomitant with neoadjuvant radiotherapy and extended to the resting period in high risk locally advanced rectal cancer. Strahlenther Onkol. 2014; 190: 158-64.

27. Xu BH, Chi P, Guo JH, Guan GX, Tang TL, Yang YH, et al. Pilot study of intense neoadjuvant chemoradiotherapy for locally advanced rectal cancer: retrospective review of a phase II study. Tumori. 2014; 100: 149-57.

28. Rodel C, Graeven U, Fietkau R, Hohenberger W, Hothorn T, Arnold D, et al. Oxaliplatin added to fluorouracil-based preoperative chemoradiotherapy and postoperative chemotherapy of locally advanced rectal cancer (the German 
CAO/ARO/AIO-04 study): final results of the multicentre, open-label, randomised, phase 3 trial. Lancet Oncol. 2015; 16: 979-89.

29. Huang CM, Huang MY, Tsai HL, Huang CW, Ma CJ, Yeh YS, et al. An observational study of extending FOLFOX chemotherapy, lengthening the interval between radiotherapy and surgery, and enhancing pathological complete response rates in rectal cancer patients following preoperative chemoradiotherapy. Therap Adv Gastroenterol. 2016; 9: 702-12.

30. Perez RO, Habr-Gama A, Sao Juliao GP, Gama-Rodrigues J, Sousa AH, Jr., Campos FG, et al. Optimal timing for assessment of tumor response to neoadjuvant chemoradiation in patients with rectal cancer: do all patients benefit from waiting longer than 6 weeks? Int J Radiat Oncol Biol Phys. 2012; 84: 1159-65

31. Habr-Gama A, Perez RO, Sao Juliao GP, Proscurshim I, Fernandez LM, Figueiredo $\mathrm{MN}$, et al. Consolidation chemotherapy during neoadjuvant chemoradiation (CRT) for distal rectal cancer leads to sustained decrease in tumor metabolism when compared to standard CRT regimen. Radiat Oncol. 2016; 11: 24 